\title{
Analysis of Muscle Activity According to the Foot Area in Stair Walking
}

\author{
Chong-hoon Lee ${ }^{1}$ and $\mathrm{Ki}$-jeong $\mathrm{Nam}^{2 *}$ \\ ${ }^{1}$ Department of Sports Science Seoul National University of Science \& \\ Technology, Seoul, Korea \\ ${ }^{2}$ Department of Physical Education Seoul National University, Seoul, Korea \\ ${ }^{1}$ leejh36@snut.ac.kr, ${ }^{2}$ rugg3@snu.ac.kr
}

\begin{abstract}
The purpose of the present study was to investigate differences the difference in muscle activity between landing with the toe side of the feet and with the entire sole during stair walking to find the landing method that has larger exercise effects. The subjects of the present study were eight adult male students of $S$ university located in $S$-si. A Noraxon electromyogram (EMG) system (Telemyo $2400 T$ system, Noraxon, USA) was used to analyze the activity of the lower extremity muscles in both motions. EMG signals were collected by attaching a bipolar surface electrode to the surface of the subject's skin. The data transmission method used was the wi-fi wireless digital communication method and the sampling rate was set to $1000 \mathrm{~Hz}$. The iEMG values of the lower extremity muscles were calculated as analytical variables and the following conclusions were drawn through analysis.

First, there was no difference in the activity of the femoral rectus muscle between the landing with the toe side of the feet and the landing with the entire sole.

Second, the landing with the toe side of the feet increased the activity of biceps femoris muscle thereby producing high exercise effects.

Third, the landing with the toe side of the feet increased the activity of the anterior tibialis and the soleus muscles thereby producing greater forces than when landing with the entire sole.
\end{abstract}

Keywords: E.M.G, walking, muscle activity

\section{Introduction}

Modern people are known to enhance their wellness through various types of regular exercise as they participate in sports activities for a considerable period of time due to economic development and the increase of leisure time. Walking is an exercise that can be easily accessed in the form of aerobic exercise that is useful for improving wellness, such as the improvement of cardiovascular function of modern people who have serious health problems due to the lack of exercise. Walking can be performed without any special skills or equipment. Walking is necessary to maintain the stability and balance, to push forward the body, and to provide basic motions necessary for movement. An appropriate range of joint motion and stability can be said to be essential for effective performance of daily living activities and safety.

Mountain climbing is a modified form of jogging and walking, which can be defined as walking over a long period on irregular terrains with high slopes, downhill roads and flatlands. ACSM (1995) reported that this type of exercise consumed the same amount energy as heavy work and caused the same extents of increases in heart rates, systolic

* Corresponding Author 
blood pressure, and myocardial oxygen consumption as those of high intensity exercise. Mountain climbing, that is, the activity to walking up and down the mountain is a very effective aerobic exercise. Many studies have shown that walking motions that account for most activities during mountain climbing use the large muscle group for long periods of time at low/medium intensities and can provide effects such as improvement of the cardiovascular system, the improvement in aerobic capacity, changes in body composition, and increases in bone density (Ohta et al., 1990; Davison et al., 1991; Duncan et al., 1991; LaCroix et al., 1996; Morris \& Hardman, 1997).

The weight loss and body fat reduction through exercise can normalize metabolic diseases associated with obesity, reduce blood lipid levels, positively affect lipoprotein metabolism, and promote health by increasing minerals in muscles and other parts of the body, reducing body fat while increasing body weight(Heywood, 2002). When aerobic exercise has been regularly performed for a long period of time, the volume of muscles increases thanks to the thickening of muscle fibers, the contents of oxygen and numerous oxidizing enzymes in muscle cells increases, the connective tissues become harder and thicker, and the total blood volume increases leading to increases in fat burning thereby reducing body fat accumulation (Horowitz, 2003).

As such, aerobic exercises such as walking are important for the prevention of adult diseases. Although mountain climbing can be said to be a good exercise with the largest amount of motions, it is not easy for most workers who work in offices. Therefore, stairway walking has recently been magnified as an aerobic exercise of workers who are quite restricted in mountain climbing or walking exercise. Stair walking is the next most frequent activity to flatland walking in everyday life while, requires more abilities than flat walking, and requires more lower-extremity force and muscle strength because the balance of body should be maintained while moving horizontally and vertically. Compared to flatland walking, stair walking shows not only increased ranges of joint motion but also larger translation and simultaneous body lifting as steps progress, which can be said to be the largest characteristics that distinguish between flatland walking and stair walking(McFadyen \& Winter, 1988). Body lifting is vertical movements of the body occurring while flexion and extension repeatedly occur in the lower extremity joints that are accompanied by many muscle activities to the extent that approximately 10-15 times of energy is necessary compared to flatland walking for the same distance (Fruin, 1991).

Diverse studies have been conducted in the field of physical education, especially on motor mechanics. The knee flexion and flexion moments in stair walking have been kinematically and motor mechanically studied in comparison with flatland walking (Jevsevar, Riley, Hodge, \& Krebs, 1993). Although diverse studies have been conducted on stairway walking in various fields, most of them focused on joint dynamics or pure activity patterns of muscles no study examined the exercise effects of stair walking. In particular, no study has been conducted at all on stair walking with landing with the toe side of the feet in place of mountain climbing exercise as a method that can produce effects similar to mountain climbing.

Therefore, the present study was conducted to compare the activity of the lower extremity muscles for landing with the toe side of the feet and that for landing with the entire sole during upward stair walking and downward stair walking based on the activity of the lower extremity muscles during general flatland walking to identify the landing method that enhances exercise effects of stair walking and recommend stair walking exercise instead of flatland walking exercise to office.

The purpose of the present study was to investigate differences the difference in muscle activity between landing with the toe side of the feet and with the entire sole during stair walking to find the landing method that has larger exercise effects. 


\section{Study Method}

\subsection{Study Subjects}

The subjects of the present study were eight adult male students of $\mathrm{S}$ university located in S-si. The characteristics of the study subjects are as shown in Table 1.

The study participants were in healthy conditions that had no musculoskeletal disease within 6 previous months with no problem in performing stairs walking. Before the present experiment, sufficient explanation was verbally given about the procedure of the experiment. Thereafter, the subjects voluntarily agreed to participate and prepared written consents before they participated in the experiment. The subjects performed motions three times in total and from among the motions; accurately performed motions were selected and analyzed.

Table 1. Characteristics of Study Subjects

\begin{tabular}{ccccc}
\hline $\begin{array}{c}\text { Number of participants } \\
\text { (person) }\end{array}$ & $\begin{array}{c}\text { Age } \\
\text { (year) }\end{array}$ & $\begin{array}{c}\text { Height } \\
(\mathrm{cm})\end{array}$ & $\begin{array}{c}\text { Weight } \\
(\mathrm{kg})\end{array}$ & $\begin{array}{c}\text { Length of lower } \\
\text { extremities }(\mathrm{m})\end{array}$ \\
\hline \hline \multirow{2}{*}{8} & $21 \pm 1.3$ & 174.38 & 74.13 & 98.31 \\
& 1 & \pm 3.81 & \pm 5.38 & \pm 2.49 \\
\hline
\end{tabular}

\subsection{Experimental Tool}

The purpose of the present study is to examine the differences in lower extremity muscle activity between the ascending and descending sections of the stair walking.

A Noraxon electromyogram (EMG) system (Telemyo 2400T system, Noraxon, USA) was used to analyze the activity of the lower extremity muscles in both motions. EMG signals were collected by attaching a bipolar surface electrode to the surface of the subject's skin. The data transmission method used was the wi-fi wireless digital communication method and the sampling rate was set to $1000 \mathrm{~Hz}$.
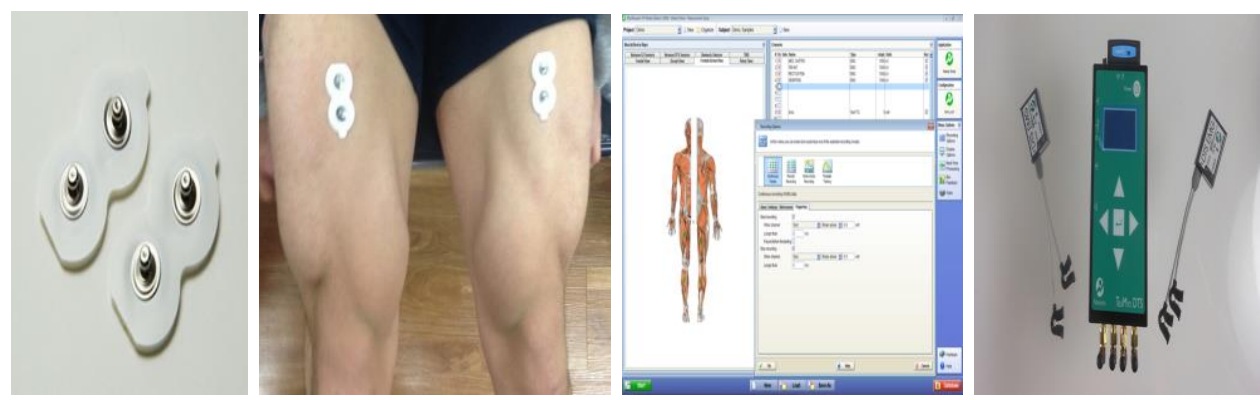

Figure 1 Electromyography 

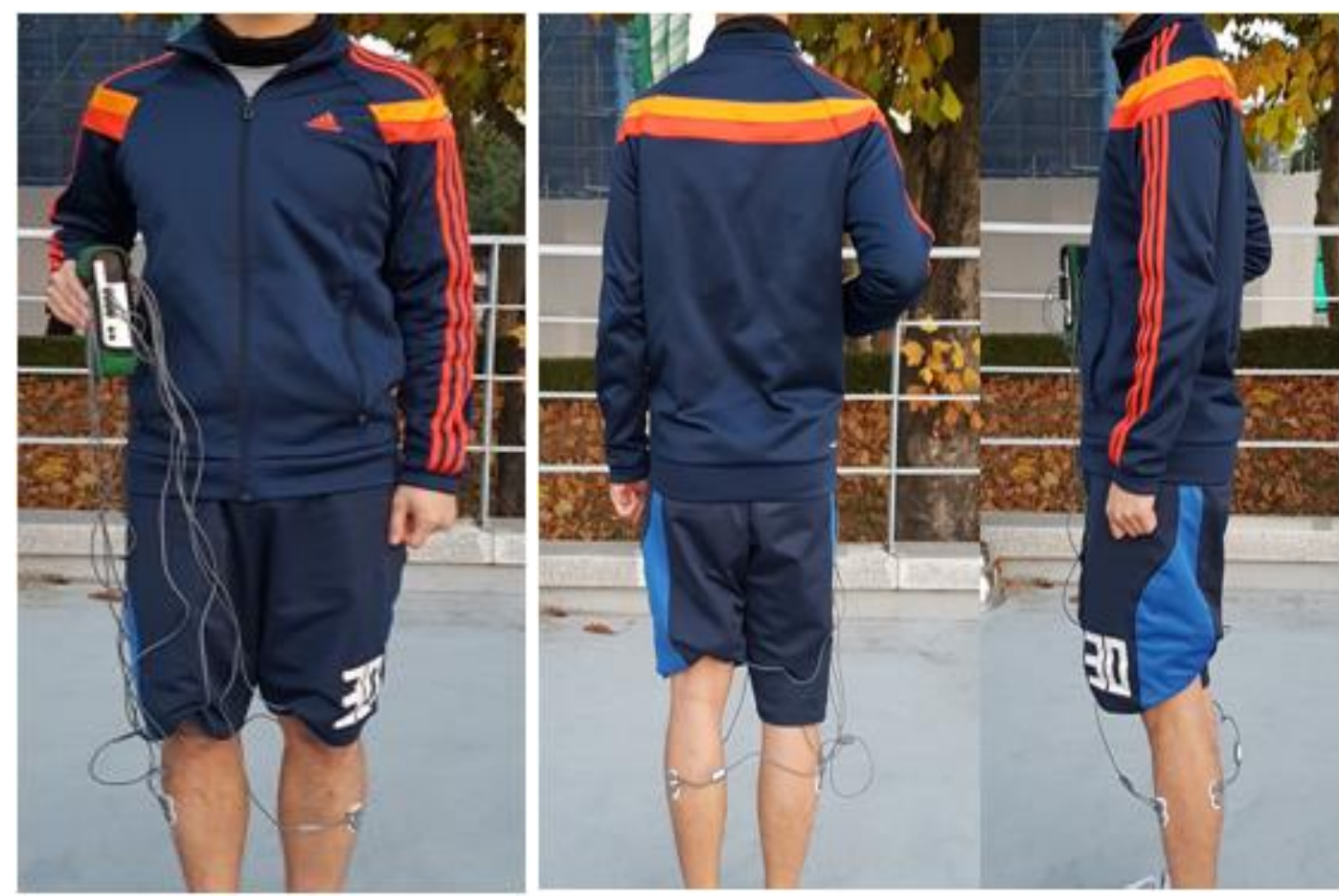

Figure 2. Views of EMG Electrodes Attached

\subsection{Experimental Procedure}

The purpose of the present study is to calculate the EMG variables of the lower extremity muscles in the landing with the toe side of the feet and landing with the entire sole during stair walking. To that end, the study participants participated in the experiment after hearing the explanation of the experiment and writing the written consent.

Before conducting the walking experiment, electrodes were attached to the lower extremity muscles of the subjects and the EMG system was prepared as shown in Figure 1. Walking was performed in three methods in total. First, in order to standardize and analyze the variables among the subjects, approximately $20-\mathrm{m}$ of flatland walking was performed Figure 3. After the flatland walking, the subjects randomly performed stair walking with landing with the toe side of the feet and stair walking with landing with the entire sole Figure 4 . The height of one stair was $16 \mathrm{~cm}$ that satisfied the standard stipulated by the law and a total of 10 stairs were used.

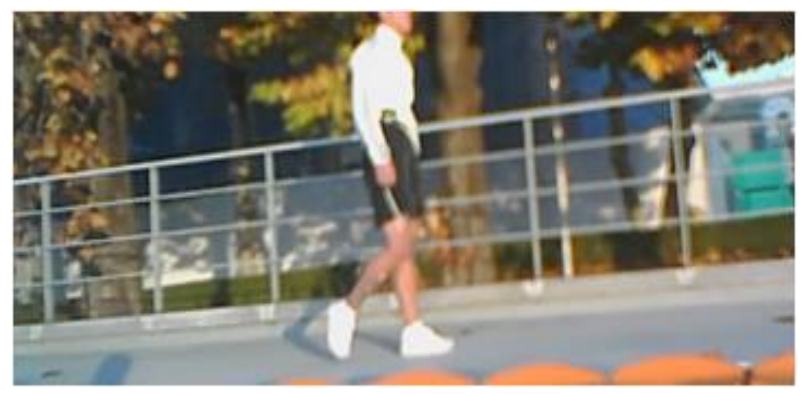

Figure 3. View of Flatland Walking 


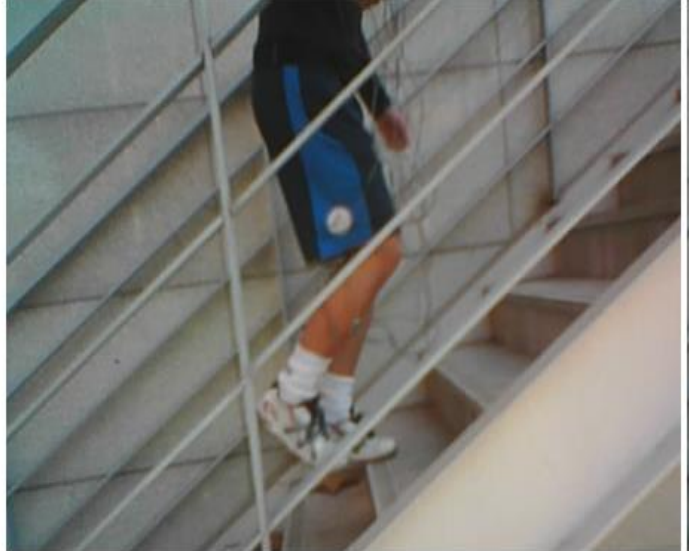

a

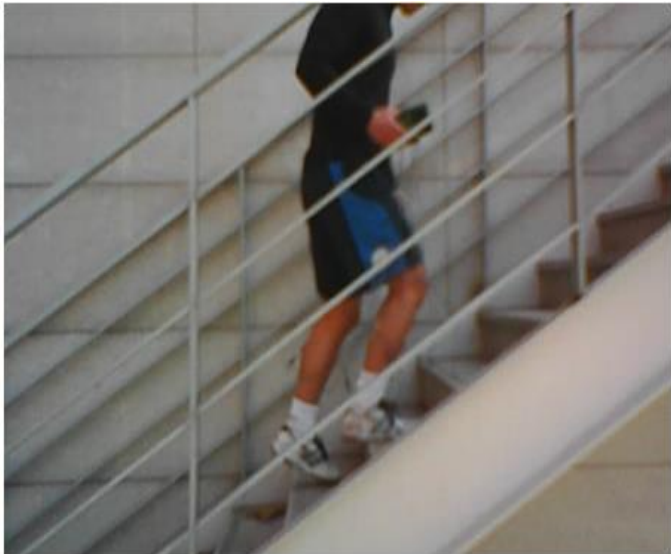

b

\section{Figure 4. a) Stair Walking with Landing with the Toe Side of the Feet, b) Stair Walking with Landing with the Entire Sole}

\subsection{Data Analysis}

The EMG data were analyzed using MR 3.4.5 program (Noraxon, USA). In the case of EMG data, the signals in the frequency band corresponding to $20-500 \mathrm{~Hz}$ were analyzed using a bandpass filter.

2.4.1. Setting of Analysis Sections: The analysis section in the three types of walking was set to a total of five stride section using one stride section defined by Perry (1992) Figure 5 .

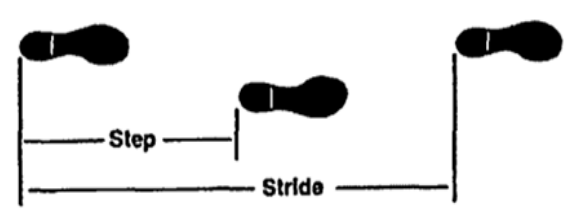

Figure 5. One Stride Section of Walking (Perry, 1992)

2.4.2. EMG Variable: In the present study, with a view to comparing the activity of the lower extremity muscles between the landing with the toe side of the feet landing with the entire sole stair walking, a total of eight muscles (femoral rectus, biceps femoris, anterior tibialis, and gastrocnemius) were selected. The muscle activity was analyzed using integral EMG (iEMG). First, full wave rectification was performed on the raw data of the EMG obtained in the experiment to prevent distortion of the quantification by negative values and to retain all the signal characteristics, and noises were removed through band filtering (Band pass filter, $20 \sim 500 \mathrm{~Hz}$ ). Finally, the iEMG was calculated by integrating the rectified signals after the removal of noises with respect to a specific time period. The calculation method of the iEMG for the specific section is as follows.

$$
i E M G=\int_{t}^{t+\tau}|E M G(t)| \cdot d t
$$

In addition, in order to compare the iEMG with the toe side landing and that with sole landing of each subject, the values in the two motions were shown as ratios and compared based on the iEMG values in general walking. 


\section{Study Results and Discussion}

\subsection{Comparison of iEMG Variables}

The results of the comparison and analysis of the ratio of the iEMG values in the two landing methods in stair ascending and descending motions in comparison with flatland walking are as shown in Table 2 and Figures 6 and 7.

Table 2. Standard Values (walking \%) of iEMG Values by Muscle

\begin{tabular}{|c|c|c|c|c|c|}
\hline \multicolumn{2}{|c|}{ Muscle position } & T-UP\% & $\mathrm{UP} \%$ & $\mathrm{~T}-\mathrm{DP} \%$ & $\mathrm{DP} \%$ \\
\hline \multirow{4}{*}{ Right } & $\begin{array}{l}\text { Femoral } \\
\text { rectus, }\end{array}$ & $631.61 \pm 505.72$ & $680.88 \pm 577.52$ & $529.43 \pm 444.38$ & $473.39 \pm 395.91$ \\
\hline & $\begin{array}{l}\text { Biceps } \\
\text { femoris }\end{array}$ & $263.91 \pm 98.44^{*}$ & $230.62 \pm 102.13^{*}$ & $160.30 \pm 89.66^{*}$ & $106.92 \pm 37.84 *$ \\
\hline & $\begin{array}{c}\text { Anterior } \\
\text { tibialis }\end{array}$ & $301.57 \pm 56.55$ & $331.15 \pm 110.03$ & $216.21 \pm 41.52^{*}$ & $169.16 \pm 13.93 *$ \\
\hline & $\begin{array}{l}\text { Soleus } \\
\text { muscle }\end{array}$ & $469.20 \pm 232.20 *$ & $282.75 \pm 126.98^{*}$ & $347.07 \pm 168.73^{*}$ & $136.66 \pm 52.87 *$ \\
\hline \multirow{4}{*}{ Left } & $\begin{array}{l}\text { Femoral } \\
\text { rectus, }\end{array}$ & $852.89 \pm 595.15$ & $882.10 \pm 778.75$ & $781.16 \pm 671.07$ & $692.81 \pm 610.88$ \\
\hline & $\begin{array}{l}\text { Biceps } \\
\text { femoris }\end{array}$ & $266.07 \pm 84.23$ & $217.61 \pm 94.43$ & $134.24 \pm 35.42$ & $94.24 \pm 24.40$ \\
\hline & $\begin{array}{c}\text { Anterior } \\
\text { tibialis }\end{array}$ & $182.83 \pm 60.57$ & $192.86 \pm 65.92$ & $101.36 \pm 60.32 *$ & $43.09 \pm 15.34^{*}$ \\
\hline & $\begin{array}{l}\text { Soleus } \\
\text { muscle }\end{array}$ & $361.68 \pm 123.93^{*}$ & $193.23 \pm 67.89 *$ & $252.52 \pm 133.02 *$ & $115.81 \pm 21.31 *$ \\
\hline & & $1 \mathrm{G}$ & *100, & $\mathrm{PiEMG/wa}$ & MG)*100 \\
\hline
\end{tabular}




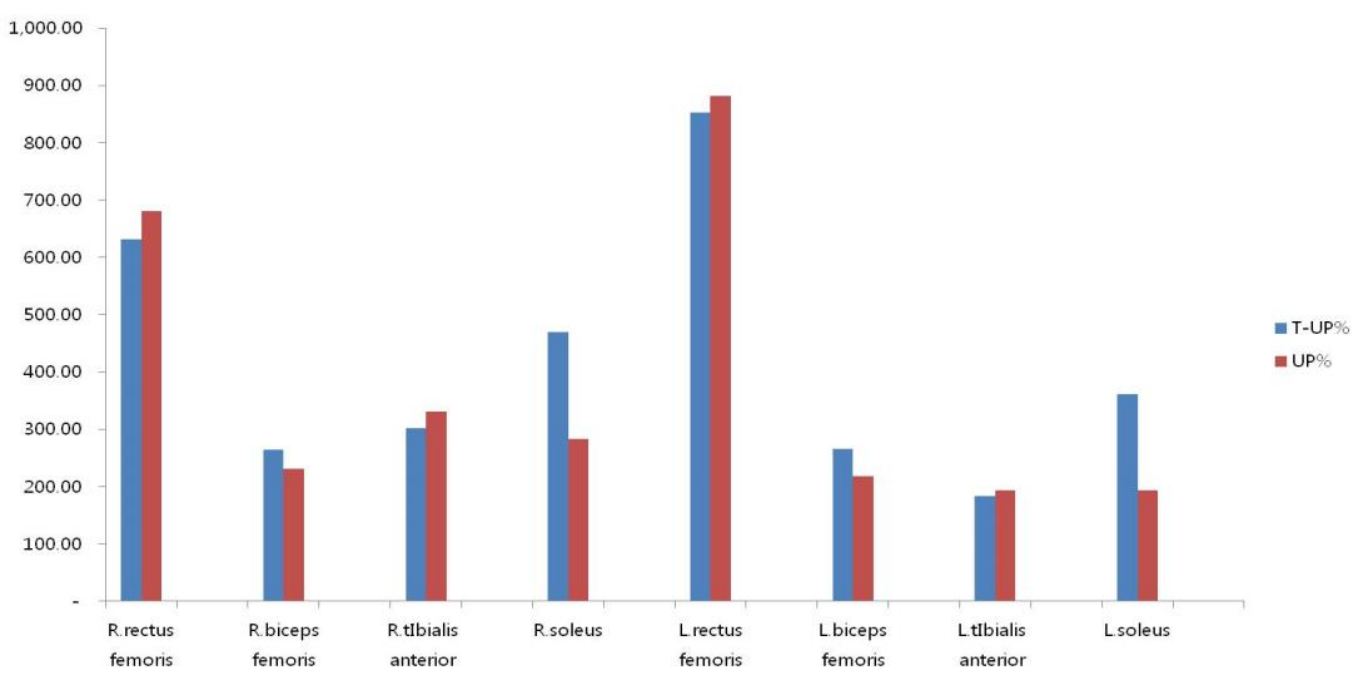

Figure 6. Muscle Activity Ratios in Two Landing Methods in Upward Stair Walking

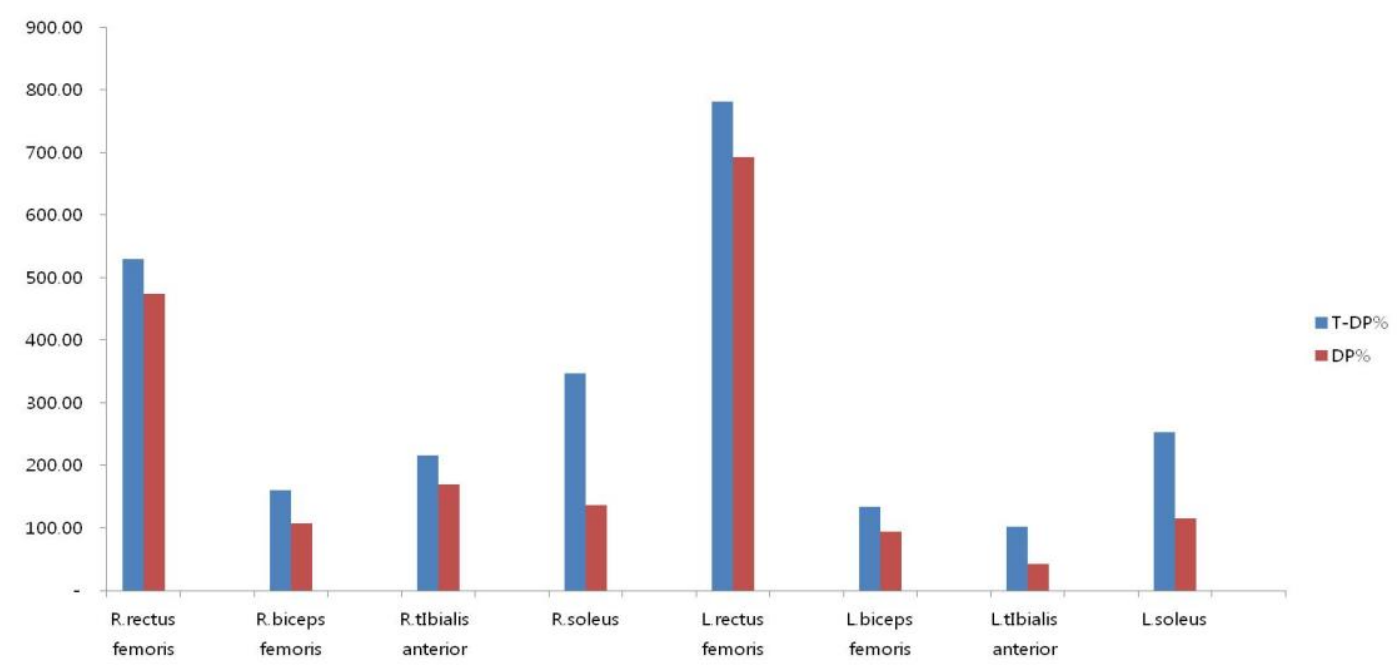

Figure 7. Muscle Activity Ratios in Two Landing Methods in Downward Stair Walking

In general, walking is a type of exercise in which the bending and extension of the knee joint are regularly performed. Studies of the lower extremity muscles mobilized in these repetitive motions can explain the gait characteristics through the scientific analysis of the walking motions.

For relative comparison of all subjects, the standardized ratio iEMG values (UP\%, $\mathrm{DP} \%$ ) based iEMG values in flatland walking were calculated as variables. The activity of the biceps femoris of the right and left femurs showed statistically significantly higher values in the landing with the toe side of the feet than in the landing with the entire sole both in upward and downward stair walking. Shinno (1971) reported that the most active activity occurs in the quadriceps femoris when during upward stair walking rather than downward stair walking and that, in both cases, hamstring activity is low. Therefore, the results of the present study indicating that the muscle activity in landing with the toe side of the feet was significantly higher are quite interesting and means that when compared to landing with the entire sole, landing with the toe side of the feet is helpful in increasing 
stimuli to the biceps femoris that is not somewhat insufficiently stimulated in general stair walking while showing similar values of the activity of other muscles.

In addition, the activity of the left and right soleus muscles showed statistically significantly higher values in the landing with the toe side of the feet than in the landing with the entire sole both in upward and downward stair walking and the activity of the left and right anterior tibialis muscles showed statistically significantly higher values in the landing with the toe side of the feet than in the landing with the entire sole both in downward stair walking only. These results mean that the landing with the toe side of the feet increases the use of the ankle during stair walking thereby producing high exercise effects. McFafyen \& Winter (1988) indicated that the femoral rectus muscle also perform meaningful activities as a knee extensor in stair walking, that during upward stair walking, the extension of the ankle helps the extension of the knee, and that to that end, the soleus muscle plays the most important role. This is consistent with the results of the present study.

On putting the results derived in the present study together, it could be seen that, although there are some differences among muscles, the activity of the muscle is about 2 to 9 times higher in stair walking than in flatland walking and that landing with the toe side of the feet increases the activity of biceps femoris, tibialis, and soleus muscle compared to landing with the entire sole while maintaining the activity of the rectus femoris. Therefore, landing with the toe side of the feet is considered necessary to higher exercise effects during stair walking.

\section{Conclusion}

The purpose of the present study was to analyze the activity of the lower extremity muscles according to landing methods during stair walking in order to find out the landing method with higher exercise effect. To that end, three types of walking: flatland walking, upward stair walking and downward walking were performed and during stair walking, the experiment was conducted in two landing methods, landing with the toe side of the feet and landing with the entire sole. The iEMG values of the lower extremity muscles were calculated as analytical variables and the following conclusions were drawn through analysis.

First, there was no difference in the activity of the femoral rectus muscle between the landing with the toe side of the feet and the landing with the entire sole.

Second, the landing with the toe side of the feet increased the activity of biceps femoris muscle thereby producing high exercise effects.

Third, the landing with the toe side of the feet increased the activity of the anterior tibialis and the soleus muscles thereby producing greater forces than when landing with the entire sole.

Based the above conclusions, stair walking is recommended as an aerobic exercise in place of mountain climbing and it can be seen that landing with the toe side of the feet increases muscle activity thereby increasing exercise effects. Future studies are considered to produce more reliable results by calculating lower extremity muscle activity during actual mountain climbing and comparing the results with lower extremity muscle activity during stair walking.

\section{References}

[1] ACSM, “ACSM's guidelines for exercise resting and prescription”, (1995), pp. 83-98.

[2] R. C. R. Davison, N. Grant, A. N. Mutrie and M. P. T. Kelly, "Walk for health?”, Journal of Sports Sci., vol. 10, (1991), pp. 556-564. 
[3] J. J. Duncan, N. F. Gordon and C. B. Scott, "Women walking for health and fitness", How much is enough? Journal of Am. Med. Associ., vol. 18, (1991), pp. 3295-3299.

[4] V. H. Heywood, “Advanced fitness assessment and exercise prescription”, Human Kinetics: Champaign, vol. 2, (2002).

[5] J. F. Horowitz, "Fatty acid mobilization from adipose tissue during exercise", Trends in Endocrinology and Metabolism, vol. 14, no. 8, pp. 386-392.

[6] H. M. Jun and J. S. Ryu, "A kinetic analysis of the lower extremity during walking on three different stair width in healthy adults", Korean Journal of Sport Biomechanics, vol. 18, no. 4, (2008), pp. 161-169.

[7] D. S. Jevsevar, P. O. Riley, W. A. Hodge and D. E. Krebs, "Knee kinematics and kinetics during locomotor activities of daily living in subjects with knee arthroplasty and in healthy control subjects", Physical Therapy, vol. 73, no. 4, (1993), pp. 229-238.

[8] A. Z. LaCroix, S. G. Leveille, J. A. Hecht, L. C. Grothaus and E. H. Wagner, "Does walking decrease the risk of cardiovascular disease hospitalizations and death in older adults?", Journal Am. Geriatric Society, vol. 44, (1996), pp. 113-120.

[9] J. N. Morris and A. E. Hardman, "Walking to health", Sports Med., vol. 23, no. 5, (1997), pp. 306-332.

[10] B. J. McFadyen and D. A. Winter, "An integrated biomechanical analysis of normal stairs ascent and descent", Journal of Biomechanics, vol. 21, (1988), pp. 733-744.

[11] R. Nisell, J. Mizrahi and J. Ekholm, "Knee and ankle Load during step-down activities", Biomechnics, X-B, Champaign, IL: Human Kinetics, (1987), pp. 1137-1142.

[12] T. Ohta, T. Kawamura, K. Hatano, M. Yokoi and U. Uozumi, "Effects of exercise on coronary risk factors in obese, middle-aged subjects", Japanese Circulation Journal, vol. 54, (1990), pp. 1459-1464.

[13] J. Perry, "Gait analysis-Normal and Pathological Function", New York, NY: McGraw-Hill, Inc., (1992).

[14] N. Shino, "Analysis of knee function in ascending and descending stairs", Med. Sport, vol. 6, (1971), pp. 202-207.

[15] D. A. Winter, "Energy generation and absorption at the ankle and knee during fast, natural and slow cadences", Clin. Orthop. Rel. Res., vol. 175, (1983), pp. 147-154.

[16] R. Kerr, G. Arnold, L. Cochrane, T. Drew and R. Abboud, "The effect of shoes on ankle injuries", Journal of Biomechanics, vol. 39, no. 33, (2006).

[17] D. C. Kerrigan, J. L. Lelas and M. E. Karvosky, "Women's shoes and knee osteoarthritis", The Lancet, vol. 357, no. 1097, (2001).

[18] W. Kim and A. S. Voloshin, "Role of plantar fascia in the load bearing capacity of the human foot", Journal of Biomechanics, vol. 28, no. 9, (1995), pp. 1025-1033.

[19] R. A. Mann, "Biomechanics of running", Symposium on the Leg, Running Sports, R. P., Mack(Ed.) St. Louis : The C.V. Mosby Co., (1980), pp. 1-29.

[20] J. C. Menant, S. D. Perry, J. R. Steele, H. B. Menz, B. J. Munro and S. R. Lord, "Effects of shoe characteristics on dynamic stability when walking on even and uneven surfaces in young and older people”, Arch Phys Med Rehabil., vol. 89, no. 10, (2008), pp. 1970-1976.

[21] B. M. Nigg and A. H. Bahlsen, "Factors influencing kinematic variables in running", In Biomechanics of Running Shoes, M. M. Nigg(ED). Champaign, III: Human Kinetics Publishers Inc., (1986), pp. 139159.

[22] B. M. Nigg and W. Liu, "The elect of muscle stiffness and damping on simulated impact force peaks during running", Journal of Biomechanics, vol. 32, (1999), pp. 849-856.

[23] B. B. Nigg, "The role of impact forces and foot pronation: a new paradigm", Clinical Journal of Sport Medicine, vol. 11, no. 1, (2001), pp. 2-9.

[24] B. Nigg, S. Hintzen and R. Ferber, "Effect of an unstable shoe construction on lower extremity gait characteristics", Clinical Biomechanics, vol. 21, (2006), pp. 82-88.

[25] D. Oeffinger, B. Brauch, S. Cranfill, C. Hisle, C. Wynn, R. Hicks and S. Augsburger, "Comparison of gait with and without shoes in children", Gait and Posture, vol. 9, (1999), pp. 95-100.

[26] R. M. Queen, A. N. Abbey, J. I. Wiegerinck, J. C. Yoder and A. N. James, "Effect of shoe type on plantar pressure: A gender comparison", Gait and Posture, vol. 31, (2010), pp. 18-22.

\section{Authors}

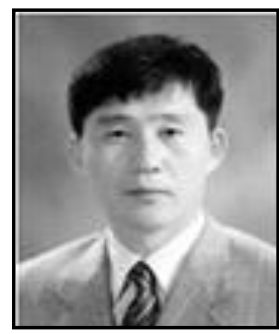

Chong-hoon Lee, he received his BS, MS in Physical Education from Seoul National University. Doctor's degree from SungKyunKwan University (School of sport science) in 1996. His major is a Sports Biomechanics. Professor, Dept. of Sports Sciences since 2000. Significant area of interest: Sport Biomechanical analysis, Development and verification of sports equipment. 


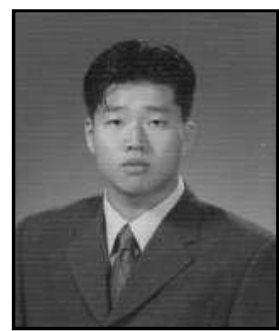

Ki-Jeong Nam, he received his BS, MS and Ph.D in Physical Education from Seoul National University. His major is a Sports Biomechanics. He is now a researcher in the Sports Biomechanics Lab at the Seoul National University and the Seoul National University of Science \& Technology. department of Biomedical Engineering. His main research interests involve studying rehabilitation, injury and efficiency. 